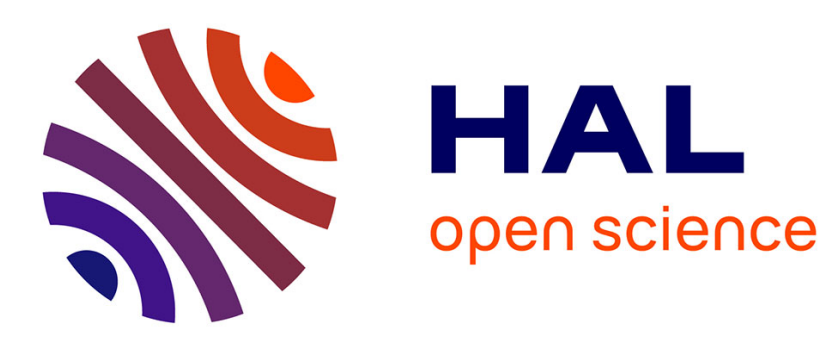

\title{
Trois modèles pour mieux comprendre la localisation des commerces de détail en milieu urbain
}

\author{
Cécile Tannier
}

\section{To cite this version:}

Cécile Tannier. Trois modèles pour mieux comprendre la localisation des commerces de détail en milieu urbain. Espace Géographique, 2003, 32 (3), pp.224-238. hal-00805094

\section{HAL Id: hal-00805094 \\ https://hal.science/hal-00805094}

Submitted on 14 Jun 2021

HAL is a multi-disciplinary open access archive for the deposit and dissemination of scientific research documents, whether they are published or not. The documents may come from teaching and research institutions in France or abroad, or from public or private research centers.
L'archive ouverte pluridisciplinaire HAL, est destinée au dépôt et à la diffusion de documents scientifiques de niveau recherche, publiés ou non, émanant des établissements d'enseignement et de recherche français ou étrangers, des laboratoires publics ou privés. 


\title{
Trois modèles pour mieux comprendre la localisation des commerces de détail en milieu urbain
}

\author{
CÉCILE TAN NIER \\ THÉMA CNRS, UMR 6049, \\ 32, rue Mégevand, 25030 Besançon cedex
}

RÉSUMÉ .- La dynamique des localisations commerciales de détail en milieu urbain est étudiée à travers la réalisation successive de trois modèles. Chacun d'eux correspond à une étape d'une démarche, dont la présentation est au cœur de cet article. Le premier modèle décrit les processus généraux à l'origine de la dynamique des localisations commerciales en milieu urbain. Le deuxième modèle consiste en l'identification de règles d'implantation des établissements commerciaux. Il permet de définir la structure du troisième modèle. Formellement le plus abouti, de par sa nature mathématique, tandis que les deux premiers sont conceptuels et graphiques, ce dernier modèle permet la simulation.

COMMERCE DE DÉTAIL, DYNAMIQUE URBAINE, LOCALISATION, MODÉLISATION, SIMULATION

ABSTRACT .- Three models for improving knowledge of urban retail location dynamics. - Three models are proposed to offer a better understanding of urban retail location dynamics. Each corresponds to one step in a methodological progression, the presentation of which is the central concern of this paper. The first step consists in modelling the general processes behind urban retail location patterns. The second step identifies the main retail location rules and defines the structure of the third model. Whereas the results of the two first steps are mainly conceptual, the mathematical nature of the last model allows for simulations.

LOCATION, MODELLING, RETAIL, SIMULATION, URBAN DYNAMICS

$0_{\text {n }}^{\text {n }}$ n s'intéresse à la localisation des établissements commerciaux de détail sédentaires, c'està-dire de tous les magasins fréquentés par la population, depuis les petits bureaux de tabac de quartier, jusqu'aux hypermarchés, auxquels s'ajoutent quelques prestataires de service exerçant en magasin (coiffeurs, pressings...). Seul le cas des villes françaises de taille comprise entre 100000 et 1000000 d'habitants est envisagé, l'hypothèse étant que les règles d'implantation des commerces sont comparables pour toutes ces villes.

L'objectif est de décrire les différentes étapes d'élaboration d'un modèle de simulation permettant d'évaluer l'attractivité des agrégats commerciaux ${ }^{1}$ d'une ville pour différents types de commerces de détail à la recherche d'un lieu d'implantation. La démarche adoptée a semblé intéressante à présenter, car la construction du modèle a été basée sur la réalisation préalable de deux autres modèles (fig. 1). Ainsi, il va être question, dans le déroulement de cet article, non

\footnotetext{
1. Par agrégat commercial (retail cluster), on entend le rassemblement de commerces en un même lieu formant un ensemble compact et présentant une certaine unité morphologique. Les établissements peuvent être alignés le long d'une ou plusieurs rues, ou réunis dans un centre commercial.
} 
du détail de la formalisation d'un modèle de simulation, mais de l'enchaînement successif de trois modèles, dont le dernier permet la simulation.

\section{Un premier modèle pour identifier les processus moteurs de l'évolution de la structure commerciale d'une ville}

Ce modèle consiste en la définition d'un système spatial, qu'on a appelé «système de localisation des établissements commerciaux de détail en milieu urbain». La figure 2 en propose une représentation graphique.

Les composants formant la structure $^{2}$ de ce système sont au nombre de quatre. Ce sont les établissements commerciaux, les lieux où ces établissements peuvent se localiser (composant la «structure commerciale»), les décideurs publics locaux (à l'origine des règlements régulant et orientant ces localisations) et la population résidentielle. Le terme d'établissement commer-

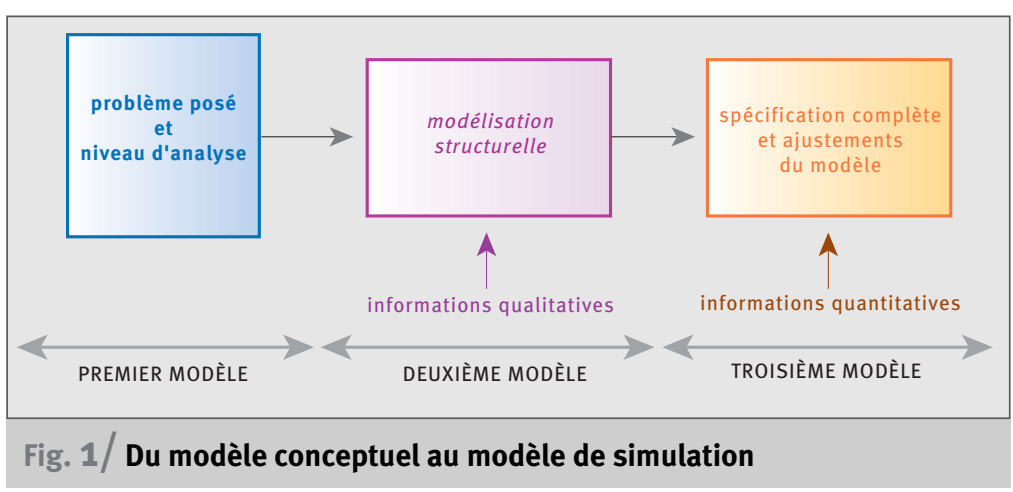
cial a été préféré à celui de commerce ou de commerçant. En effet, sa neutralité permet de rassembler sous une même dénomination tous les acteurs "commerçants", quelle que soit la forme de commerce pratiquée. En outre, il représente bien la réalité physique de l'acte de s'implanter et permet d'éviter les problèmes d'identification liés aux entreprises possédant plusieurs établissements. L'évolution de la structure commerciale d'une ville est le résultat de processus complexes d'interactions entre les différents composants du système. La multiplicité de ces interactions permet le maintien de la structure du système.

\section{Interactions entre les acteurs}

Les acteurs économiques sont tenus de se conformer aux décisions des acteurs publics (respect des lois), tandis que les décideurs publics doivent émettre des règlements permettant la bonne marche des établissements commerciaux. L'élection (directe ou indirecte) des décideurs publics par les acteurs économiques est garante de ce bon fonctionnement. Il est également nécessaire que les établissements commerciaux vendent des biens que les consommateurs (c'est-à-dire la population résidentielle) désirent et soient en mesure d'acheter.

\section{Interactions entre les acteurs et le territoire}

Au-delà du maintien de la structure du système, ce type d'interactions lui est inhérent. Il caractérise en effet un système spatial. Les acteurs publics, à travers les documents d'urbanisme (plans d'occupation des sols, schémas directeurs d'aménagement urbain...) et les projets d'aménagement urbain (création de zones d'activité, construction de nouvelles voies de communication, création de zones piétonnières en centreville, transformation des plans de circulation...), influencent le devenir de la structure commerciale d'une ville. Les établissements commerciaux, par leurs ouvertures, fermetures et changements de localisation, font de même. Mais si les acteurs modifient

2. Le terme "structure" est employé dans cet article selon deux acceptions.

La structure d'un système est l'organisation des parties de celui-ci qui lui donne sa cohérence et qui en est la caractéristique permanente ( $d^{\prime}$ après le Petit Larousse de 1996).

En revanche, dans l'expression "structure urbaine" ou "structure commerciale", ce terme désigne la manière dont les éléments sont disposés les uns par rapport aux autres, cette configuration évoluant le plus souvent au cours du temps. 


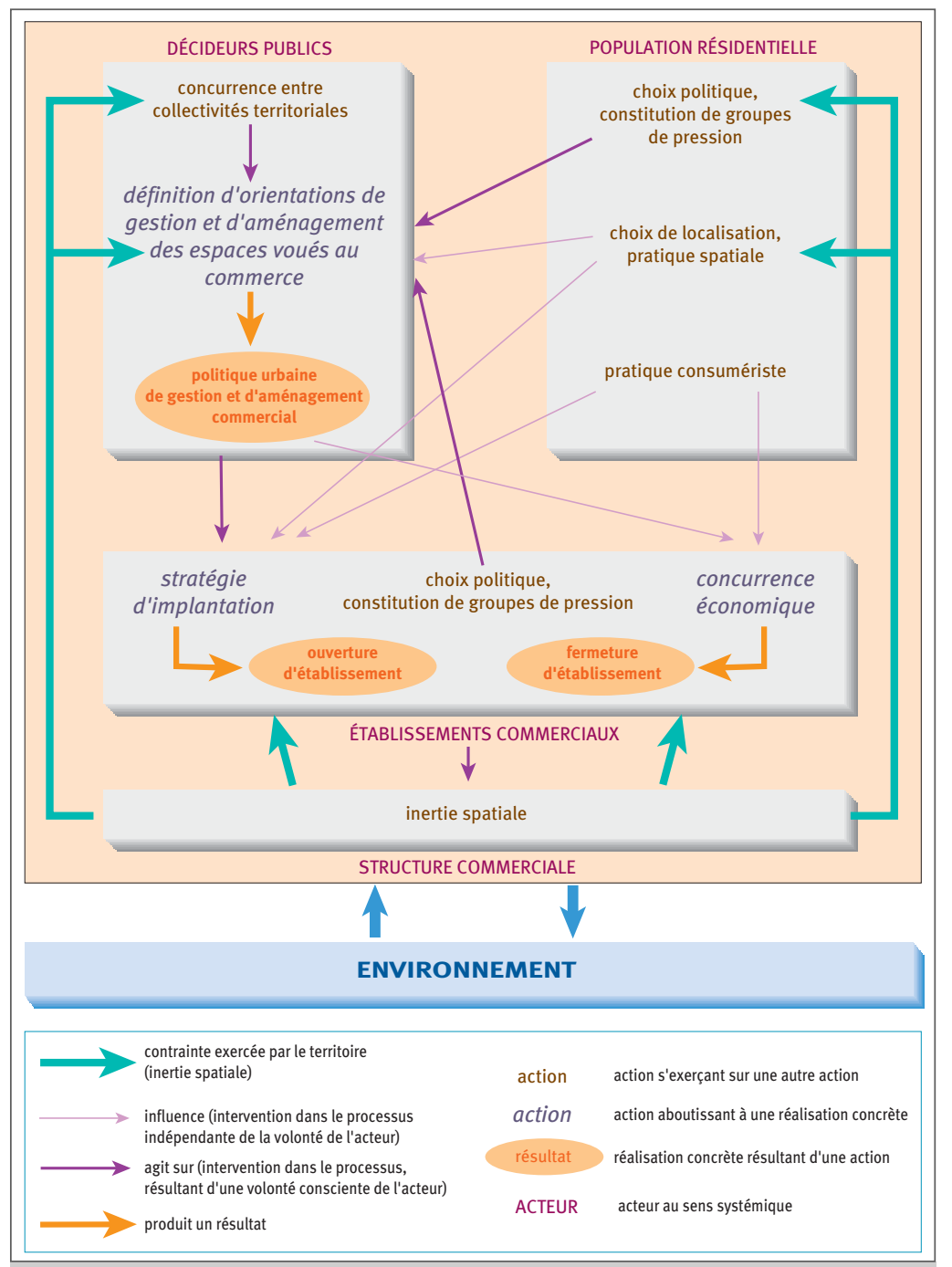

Fig. 2 / Le système de localisation des établissements commerciaux de détail en milieu urbain le tissu commercial, celui-ci influence également le comportement des acteurs. Par exemple, l'implantation d'un hypermarché dans un agrégat commercial a bien souvent pour conséquence l'installation dans son sillage d'autres établissements commerciaux (grandes et moyennes surfaces spécialisées notamment). De même, au moment du choix d'un lieu de résidence, la présence de commerces de proximité représente un élément attractif non négligeable.

\section{Interactions entre le système et son environnement}

Un bon fonctionnement du système suppose des emplois pour que les habitants puissent devenir des consommateurs potentiels, des infrastructures de transport pour permettre aux consommateurs potentiels de se rendre dans les établissements commerciaux et devenir des acheteurs effectifs, des infrastructures de commerce de gros pour alimenter les établissements commerciaux de détail... Ainsi, le système de localisation des établissements commerciaux de détail en milieu urbain interagit de façon intense avec son environnement. Dans le sens de l'action de l'environnement sur le système de localisation des établissements commerciaux, on peut citer l'impact du réaménagement des centres-villes (création de zones piétonnières notamment) sur l'offre commerciale de ces quartiers (Metton, 1984) ou l'influence du tissu urbain sur le fonctionnement commercial. Par exemple, dans le cas d'implantations commerciales le long d'une rue, une rupture du linéaire commercial par des habitations ou d'autres équipements est préjudiciable. Si ces ruptures se multiplient, l'ensemble du linéaire est, à court terme, menacé. Un tissu urbain très structuré permet donc le renforcement de l'armature commerciale et évite le mitage de l'espace. En sens inverse, on observe la création de grands centres commerciaux en Île-de-France pour restructurer l'agglomération parisienne (Metton, 1984), l'organisation de zones piétonnières de centre-ville autour de l'activité commerciale.

Outre l'environnement proche du point de vue spatial (la ville considérée), le contexte social et économique régional, national et international influence aussi le 
fonctionnement du système (législation nationale concernant les implantations commerciales, stratégie et organisation des grands groupes de distribution, situation de la ville dans un système de villes...). Et, si l'évolution des localisations commerciales correspond au volet spatial de la dynamique de l'offre commerciale d'une ville, les phénomènes relatifs à la dynamique non spatiale interviennent aussi dans les choix d'implantation des établissements: évolution des formes de vente, évolution de l'offre en magasin, évolution démographique et sociale des acteurs économiques du commerce (chefs d'entreprise, salariés...), lesquels doivent également être considérés comme faisant partie de l'environnement du système.

Parmi les quatre composants formant la structure du système, trois sont des composants "acteurs", le quatrième étant un composant "territoire». D'un point de vue systémique, un acteur est un composant d'un système qui déclenche une action, voire une interaction. En ce sens, les décideurs publics, les établissements commerciaux, la population résidentielle, mais aussi le territoire sont des acteurs du système de localisation des établissements commerciaux de détail. Cependant, le territoire est un acteur particulier, son seul mode d'action étant son inertie.

Il est intéressant de remarquer qu'un même individu, selon les circonstances, peut être soit acteur, soit agent. Les agents n'agissent pas directement sur le fonctionnement d'un système; ils influencent le comportement d'autres acteurs (leurs pratiques spatiales, leurs préférences sont prises en compte par ces derniers pour définir leurs stratégies), sans que cette action constitue une volonté consciente de leur part. Leur rôle est essentiellement passif. Sur la figure 2, la différenciation des flèches selon leur signification ("agit sur» ou «influence») exprime cette distinction entre acteurs et agents. Ainsi, parmi les acteurs (au sens large) du système de localisation des commerces en milieu urbain, la population résidentielle n'est acteur (au sens strict, s'opposant à agent) que lors de la constitution d'un groupe de pression et au moment de l'expression de ses choix politiques.

Deux réalisations concrètes découlent du fonctionnement du système de localisation des établissements commerciaux. La première consiste en la mise en place de politiques de gestion et d'aménagement des espaces liés au commerce. Elle est le résultat de deux jeux d'acteurs: les conflits d'intérêts entre acteurs sociaux, économiques et politiques concernant la gestion de l'espace urbain, d'une part, et, d'autre part, la concurrence entre collectivités locales pour attirer sur leur territoire des établissements commerciaux. L'autre réalisation concrète résultant du fonctionnement du système est l'ouverture et la fermeture d'établissements commerciaux. Elles seules sont directement à l'origine d'une transformation de la structure commerciale d'une ville. Elles résultent de deux actions, les stratégies d'implantation des établissements commerciaux de détail et la concurrence économique à laquelle ils se livrent une fois installés.

Finalement, les jeux d'acteurs, internes au système de localisation des commerces et aboutissant à une réalisation concrète, consistent en quatre processus de concurrence.

\section{Les stratégies d'implantation des établissements commerciaux, manifestation d'un processus de concurrence pour l'occupation du sol}

Les stratégies d'implantation des établissements commerciaux peuvent être considérées comme la manifestation d'un processus de concurrence pour l'occupation du sol, dans le sens où chaque établissement cherche à obtenir le meilleur emplacement possible. 
Deux types d'acteurs interviennent dans le champ des implantations commerciales: les établissements commerciaux de détail et les décideurs publics participant à la définition d'une politique d'aménagement et de gestion de la structure commerciale du territoire urbain (élus municipaux essentiellement). Les résidents d'une agglomération représentent dans ce cadre non pas un groupe d'acteurs, mais d'agents.

L'étude des stratégies d'implantation a pour but de comprendre où les établissements vont s'installer, sans estimer leur risque de fermeture. Ce faisant, on n'évalue pas directement la rentabilité potentielle des magasins, qui dépend des parts de marché des concurrents et de la capacité d'achat de la population de l'aire de chalandise. Cet aspect de la question est implicitement pris en compte à travers la comparaison de l'attractivité de différents noyaux commerciaux sur les établissements, sachant toutefois que des emplacements très attractifs peuvent être caractérisés par des taux de rotation des établissements élevés.

\section{Concurrence entre acteurs sociaux, économiques et politiques} concernant la gestion du territoire urbain

Les acteurs socio-économiques souhaitent, tous, le bon fonctionnement ${ }^{3}$ de leur lieu d'implantation. Mais il arrive que le bon fonctionnement souhaité par les acteurs dominants d'un lieu soit en contradiction avec celui souhaité par d'autres acteurs de ce lieu ou par ceux d'un autre lieu. Les deux groupes exercent alors des pressions sur les institutions publiques, soit directes (par exemple, pressions du lobby des commerçants de centre-ville pour interdire la création d'une nouvelle zone d'activité commerciale, demande sociale émanant d'un comité de quartier pour subventionner la restructuration de leur centre commercial...), soit indirectes (élection des représentants politiques).

Dans tous les cas, c'est le décideur public qui donne suite ou non aux réclamations: s'il décide d'agir, il va introduire de nouvelles contraintes dans le processus de compétition pour l'occupation du sol. Les conflits d'intérêts territoriaux entre acteurs sociaux et économiques influencent donc directement les ouvertures d'établissements commerciaux.

Dans le cadre de ce processus, les résidents d'une agglomération, d'agents, peuvent devenir acteurs, par exemple lors de la constitution d'un groupe de pression visant à faire aboutir une demande sociale telle que le maintien d'un commerce de proximité. Deux autres acteurs entrent aussi en jeu: les décideurs publics et les acteurs économiques du commerce. Concernant ces derniers, il apparaît plus approprié de considérer les personnes physiques que les établissements commerciaux. En effet, les acteurs économiques du commerce, lorsqu'ils cherchent à faire valoir leurs idées concernant la gestion et l'aménagement du territoire urbain agissent, soit de façon isolée (c'est souvent le cas des grands distributeurs), soit en groupe (au sein d'une union des commerçants par exemple).

\section{Concurrence entre collectivités locales pour attirer sur leur territoire} des établissements commerciaux

3. II est sous-entendu que, pour un acteur, un bon fonctionnement est celui qui va dans le sens de ses intérêts.
Le cas des zones d'activités périphériques est à ce sujet exemplaire. La plupart sont en effet valorisées par des atouts équivalents: desserte autoroutière, proximité d'une agglomération importante... Ce qui les place d'ores et déjà dans une situation très concurrentielle. Or, les collectivités locales accentuent cette concurrence par leur 
propre concurrence pour attirer sur leur territoire des établissements commerciaux. Celle-ci est encore exacerbée par la multiplicité des acteurs qui interviennent sur le terrain, dont chacun possède sa logique propre et son échelle de stratégie (communes, chambres de commerce et d'industrie, département, région...).

Les acteurs de cette concurrence sont les collectivités locales, tandis que les établissements commerciaux en sont les agents.

\section{Concurrence économique entre établissements commerciaux, dans le but d'assurer leur survie}

Un établissement, pour espérer se maintenir, doit répondre le mieux possible aux attentes de la clientèle potentielle. Ceci implique de prendre en compte les caractéristiques culturelles et l'évolution des modes de consommation de la population, autrement dit, certains aspects de la dynamique non spatiale de l'offre commerciale. Les acteurs de ce processus sont les établissements commerciaux, tandis que les consommateurs en constituent le vecteur (les établissements se concurrencent à travers les consommateurs) et doivent donc, dans ce cas, être considérés comme des agents.

La conception d'un premier modèle a permis de mettre en évidence les processus généraux à l'origine de la dynamique des localisations commerciales d'une ville. Ceci va servir de point de départ à la réalisation du modèle suivant.

\section{Un deuxième modèle pour identifier les règles d'implantation des établissements commerciaux de détail en milieu urbain}

Il aurait été envisageable de chercher à mettre en évidence les stratégies d'implantation de différents types de commerces. Mais les choix de localisation des établissements commerciaux résultent de comportements décisionnels pour l'essentiel non rationnels et, qui plus est, très divers quant à leur niveau de rationalité dans la non-rationalité. C'est pourquoi, plutôt que d'étudier les stratégies des établissements, il est apparu plus profitable de chercher à mettre en évidence les règles régissant l'implantation des commerces en ville. Il s'agit alors de déterminer à quelles combinaisons de caractéristiques du territoire correspondent quelles implantations préférentielles (petits commerces de proximité, hypermarchés, maxi-discomptes...). Ceci n'implique ni de connaître les niveaux de rationalité des différents types d'établissements, ni de leur affecter un unique niveau de rationalité (parfaite ou limitée) forcément éloigné de la réalité.

C'est à un aspect du fonctionnement général du système de localisation des établissements commerciaux de détail en milieu urbain que nous nous intéressons maintenant en abordant l'étude des règles d'implantation des établissements commerciaux. Nous nous concentrons désormais sur un type d'acteur, les établissements commerciaux de détail, et un type d'action, les ouvertures d'établissements. Il est ici intéressant de remarquer que les mots les plus couramment employés conviennent bien pour qualifier ces deux points de vue sur la dynamique spatiale de l'offre commerciale: le mot «implantation" rappelle l'acte de s'implanter et donc l'ouverture de commerces, tandis que celui de «localisation» est plus général et recouvre l'ensemble des configurations spatiales résultant des ouvertures et des fermetures d'établissements.

Comme ce deuxième modèle a été élaboré en vue de la réalisation d'un troisième modèle devant permettre d'évaluer l'attractivité des agrégats commerciaux d'une ville, 
4. Une description détaillée de chaque critère (énoncé précis, références bibliographiques dans lesquelles il est mentionné, remarques éventuelles) est fournie dans les annexes II de Tannier, 2000. les mécanismes qui permettent d'expliquer pourquoi, au sein d'un même agrégat commercial, certaines boutiques s'installent plus volontiers à proximité de certaines autres ou dans certains emplacements privilégiés (coins de rue, espaces esthétiquement cotés...), ne sont pas pris en compte.

Définir des règles d'implantation des commerces en milieu urbain consiste à établir une série de relations entre deux ensembles d'éléments. Le premier ensemble regroupe tout ce qui relève de l'acteur "établissement commercial» lui-même, son comportement, ses aspirations en matière de choix de localisation, indépendamment des autres éléments du système. Il s'agit donc d'un aspect interne au composant "établissements commerciaux». Le deuxième ensemble correspond à l'influence des autres parties du système sur les choix de localisation des établissements commerciaux, à savoir les composants "structure commerciale", "population résidentielle", "décideurs publics", "établissements commerciaux" hors stratégies de localisation (effets de la concurrence économique, rôle des associations commerçantes...) et l'environnement du système. Plus précisément, ce deuxième ensemble correspond aux éléments du système de localisation des commerces qui sont susceptibles de différencier les uns des autres les agrégats commerciaux d'une ville, au regard d'un établissement commercial de détail quelconque cherchant à s'implanter. De ce fait, de l'environnement du système, on ne considère que les éléments relatifs à l'espace urbain (contexte socio-économique national et international, autres espaces urbains en relation avec l'espace considéré...). De même, de la structure commerciale d'une ville, on ne retient que les éléments relevant du niveau d'analyse de l'agrégat.

Ainsi, nous cherchons à établir une série de relations entre un premier ensemble regroupant les caractéristiques des établissements commerciaux, qui différencient ces derniers quant à leurs aspirations concernant leur lieu d'implantation, et un deuxième ensemble, regroupant les critères qui différencient les agrégats commerciaux d'une ville au regard d'établissements commerciaux à la recherche d'un lieu d'implantation.

Pour établir la liste des critères de différenciation des agrégats commerciaux d'une ville, une centaine d'écrits ont été étudiés, dans lesquels les auteurs présentent, ou évoquent de façon plus indirecte, les critères qui, pour eux, entrent en jeu dans le choix d'un lieu d'implantation par un établissement commercial. Ces écrits peuvent être ventilés en deux catégories: à destination, soit d'un public scientifique (géographes, sociologues, économistes universitaires ou travaillant dans divers organismes de recherche), soit de praticiens (commerçants et cadres commerciaux, cadres des services techniques de collectivités locales, chargés d'étude d'une agence d'urbanisme...). De cette recherche bibliographique, cinquante-huit critères ont été relevés 4 .

Ainsi, l'entité spatiale de référence est l'agrégat commercial et les cinquante-huit critères relevés dans la littérature en représentent les attributs (au sens informatique du terme). Il a été décidé de classer ces critères en six catégories, tenant compte à la fois du rôle de chacun d'eux dans le système de localisation des commerces, et de la distinction entre le niveau de représentation des phénomènes (c'est-à-dire l'entité spatiale de référence) et leur niveau d'analyse (échelle spatiale à laquelle on peut observer ces phénomènes) (fig. 3).

1. Caractéristiques de l'agrégat commercial.-Cette catégorie comprend les critères décrivant la structure commerciale de l'agrégat, à l'exception de ceux caractérisant les emplacements disponibles, ainsi que tous les éléments de l'environnement directement liés à l'agrégat, quel que soit leur niveau d'analyse. 
2. Caractéristiques des emplacements disponibles. - Les critères appartenant à cette catégorie auraient logiquement dû être considérés comme faisant partie des caractéristiques de l'agrégat commercial. Mais leur nombre, leur variété et l'importance qu'ils revêtent, quant aux choix de localisation, justifiaient la création d'une catégorie qui leur soit propre.

3. Caractéristiques de la clientèle de l'agrégat commercial.- Tous les critères décrivant la population résidentielle appartiennent à cette catégorie, quelle que soit l'échelle à laquelle ils doivent être observés. Il est intéressant de remarquer que les critères relevant d'un niveau d'analyse global décrivent une clientèle de rayonnement, tandis que ceux qui relèvent d'un niveau d'analyse par quartier décrivent une clientèle de proximité.

4. Caractéristiques du quartier dont fait partie l'agrégat commercial.- Ces critères relèvent du niveau d'analyse global et du niveau d'analyse par quartier. Ils représentent l'influence de l'environnement, dans ses composants historiques, urbanistiques, morphologiques et topographiques, sur le système des localisations commerciales.

5. Accessibilité de l'agrégat. - Ces critères font l'objet d'une catégorie à part entière car on les considère comme un ensemble d'éléments qui modulent l'intensité des échanges potentiels entre les consommateurs et les établissements commerciaux.

6. Interventions des acteurs publics et économiques sur l'agrégat commercial.- Parmi ces critères, les quatre premiers décrivent le contexte du choix de localisation, mais ne peuvent être considérés comme des critères de différenciation des agrégats proprement dits. Le niveau d'analyse des phénomènes est très varié et rarement univoque. Par exemple un projet urbain intégrant toutes les composantes urbaines peut concerner soit une ville dans son ensemble, soit un quartier, soit une portion de quartier.

De l'abondante bibliographie dépouillée, il ressort également que les établissements font varier leurs stratégies de localisation en fonction des modalités de dix caractéristiques.

1. Commerce de rayonnement ou de proximité.- Une activité commerciale de proximité induit des déplacements sur une courte distance, généralement au sein d'un même quartier, tandis que les commerces de rayonnement recrutent leur clientèle sur un territoire beaucoup plus vaste (déplacements intra-urbains et interurbains).

2. Fréquence d'achat des produits vendus. - Elle peut être quotidienne, hebdomadaire ou plus épisodique. Elle varie principalement selon qu'il s'agit d'un commerce de rayonnement ou de proximité.

3. Attractivité intrinsèque du magasin. - Il s'agit du pouvoir d'attraction d'un commerce sur la clientèle, indépendamment de l'attraction exercée par le noyau commercial dont fait partie ce commerce. Les «locomotives» en particulier (gros hypermarchés, grandes surfaces spécialisées telles que Kiabi, Castorama) sont des établissements bénéficiant d'une forte attractivité intrinsèque. C'est aussi le cas des maxi-discomptes et de certains magasins particulièrement réputés ou très spécialisés.

4. Standing des produits vendus.- Les produits vendus peuvent être de qualité et de coût très divers, depuis les produits caractérisés par un prix de vente très faible jusqu'aux produits de grand luxe.

5. Segment de clientèle visée.- Outre le standing du magasin, un magasin peut cibler des types de clientèle spécifiques (étudiants, touristes...) ou, au contraire, tout type de clientèle. 
1. Caractéristiques de l'agrégat commercial

- nombre et type de magasins présents

- présence d'établissements « locomotives»

- nom des enseignes présentes

- qualité de la présentation des magasins

- surface de vente totale de l'agrégat

- type d'agrégat commercial (rue commerçante, centre commercial, galerie commerciale, quartier commerçant...)

- facilités de parking de l'agrégat

- situation de l'agrégat par rapport aux circuits de distribution

- localisation de l'agrégat sur une ou plusieurs communes

- estimation du chiffre d'affaires réalisé par les établissements

\section{Caractéristiques des emplacements disponibles}

- nombre et taille des espaces (terrains ou locaux) disponibles

- caractéristiques des locaux disponibles (de plain-pied ou avec étages, hauts ou bas de plafond...)

- offre immobilière

- prix fonciers, loyers

- taxes foncières

- droit au bail

\section{Caractéristiques de la clientèle de l'agrégat commercial}

Niveau d'analyse global

- nombre d'habitants et composition sociale de la population de l'aire de chalandise

- taux de croissance de la population de l'aire de chalandise

- caractéristiques culturelles de la population de l'aire de chalandise

- revenus de la population de l'aire de chalandise

- pouvoir d'achat de la population de l'aire de chalandise

- clientèle potentielle totale de l'agrégat commercial

- attractivité de l'agrégat commercial sur la population non résidente

- pratiques spatiales des consommateurs

- estimation de la demande des ménages

Niveau d'analyse par quartier

- nombre d'habitants et composition sociale de la population du quartier

- existence d'un marché spécifique

Niveau d'analyse infra quartier

- fréquentation effective de l'agrégat commercial

- fréquentation de l'agrégat commercial par de la clientèle piétonne
4. Caractéristiques du quartier dont fait partie l'agrégat

\section{Niveau d'analyse global}

- situation du quartier dans la ville

- rôle du quartier dans la ville (animation, centralité...)

- poids de la tradition, prestige du quartier

- présence d'activités autres que commerciales

Niveau d'analyse par quartier

- (in)sécurité du quartier

- image du quartier

- spécificité du quartier

- particularismes topographiques du quartier

- standing du quartier

\section{Accessibilité de l'agrégat}

Niveau d'analyse global

- accessibilité pour les automobiles

- accessibilité grâce aux transports publics (notamment métro et tramway)

- multimodalité des déplacements (piétons, automobiles, transports en commun) plus ou moins favorisée

- qualité de l'accès pour les livraisons

Niveau d'analyse par quartier

- accessibilité à la ou aux routes principales

- qualité de l'accès aux rues commerçantes

- qualité de l'entrée et de la sortie du noyau commercial

\section{Niveau d'analyse infra quartier}

- qualité des circulations internes (automobile et piétonne)

- lisibilité de l'agrégat, bon fléchage des magasins

- niveau de congestion du trafic

\section{Interventions des acteurs publics et économiques sur l'agrégat}

- lois et règlements d'urbanisme

- politiques d'aménagement urbain

- existence d'un projet urbain intégrant toutes les composantes urbaines

- existence ou non d'une procédure de requalification de l'espace urbain

- qualité de la concertation (voire du partenariat) entre les acteurs publics et économiques

- action des groupements de commerçants

- heures et jours d'ouverture des commerces

- animation du lieu permanente, périodique ou plus occasionnelle

Fig. 3 / Les critères de différenciation des agrégats commerciaux

6. Poids et encombrement des produits vendus. - Le critère de distinction majeur est la possibilité ou non de transporter ses achats à pied.

7. Nature des produits proposés.- Ceux-ci peuvent être soit des biens d'achat courant (alimentation générale, cigarettes...), soit des biens d'achat impulsif (bonbons, bijouterie fantaisie...), ou encore des biens d'achat programmé (meubles, électroménager...).

8. Taille du magasin. - Les magasins de grande taille sont destinés à la vente soit d'un grand nombre de produits à un grand nombre de personnes, soit d'un moins grand nombre de produits très encombrants à un nombre variable de personnes. 
9. Établissement indépendant ou non.- On distingue, d'un côté, les commerces indépendants et de l'autre, les magasins franchisés ou en partenariat et les établissements succursalistes.

10. Montant du capital investi-_ La mise de fonds initiale varie notablement selon qu'il s'agit d'un entrepreneur indépendant ou d'une chaîne de magasins.

Nous connaissons maintenant le contenu des deux ensembles dont nous souhaitons identifier les relations. Il s'agit alors d'analyser ce contenu afin de ne conserver que les éléments réellement nécessaires à la poursuite du travail.

Parmi les dix caractéristiques des établissements, sept suffisent à différencier ces derniers les uns des autres. La caractéristique «fréquence d'achat des produits» peut être éliminée car elle apporte les mêmes informations sur les choix de localisation des établissements que la caractéristique "de rayonnement ou de proximité». Il en est de même pour la caractéristique "poids et encombrement des produits", qui a les mêmes conséquences sur les choix de localisation que la «taille du magasin». Le "montant du capital investi au départ" varie principalement selon le niveau de standing du magasin et l'appartenance ou non à une entreprise possédant plusieurs filiales ou à un réseau de franchise. On peut donc également l'éliminer de la liste.

Les critères de différenciation des agrégats commerciaux étant beaucoup plus nombreux, la sélection de ceux réellement utiles n'est pas aussi simple. On commencera par remarquer que le choix du meilleur site d'implantation se fait en fonction d'un ensemble d'objectifs et de contraintes. On peut considérer que les établissements commerciaux ne tiennent compte que de leurs objectifs pour évaluer l'attractivité des agrégats commerciaux d'une ville et que les contraintes interviennent en second lieu, au moment du choix final d'un lieu d'implantation. Dès lors, si on adopte le raisonnement suivant: "un objectif peut toujours être revu à la baisse, par n'importe quel établissement, ce qui n'est pas le cas d'une contrainte", il s'avère que, parmi les cinquante-huit critères, seuls deux d'entre eux représentent de réelles contraintes pour le choix d'un site par un établissement. Il s'agit des lois et règlements d'urbanisme (plan d'occupation des sols, loi Raffarin...) et du nombre et de la taille des espaces (terrains ou locaux) disponibles. D'une part, les lois et les règlements d'urbanisme sont (théoriquement) incontournables, d'autre part, tout établissement a besoin d'un emplacement d'une taille minimum pour s'installer. L'aspect financier (prix fonciers, loyers, droit au bail) peut a priori sembler être une contrainte, mais dans les faits, il s'avère que les obstacles financiers sont rarement totalement insurmontables. On mentionnera ici que, sur la figure 2, les flèches "agit sur» et "contrainte exercée par le territoire», aboutissant toutes deux à l'élément "ouverture d'établissement", s'expliquent par l'existence de ces deux contraintes liées à l'ouverture d'établissements commerciaux.

Conserver uniquement les critères qui représentent des objectifs ne permet finalement d'en éliminer que deux. Parmi les cinquante-six critères restants, n'ont été retenus que ceux pour lesquels il est possible d'identifier un lien avec une ou plusieurs caractéristiques descriptives des établissements. Leur sélection se fait en fonction des conditions suivantes: les critères doivent être en cohérence avec le niveau de finesse de l'analyse; il nous faut connaître les modalités selon lesquelles ils déterminent l'attractivité d'un agrégat commercial; ils doivent jouer différemment sur l'attractivité d'un agrégat selon le type d'établissement. En vue de la réalisation ultérieure d'un modèle de simulation, on choisira, parmi plusieurs critères redondants, le plus facile d'entre eux à renseigner. 


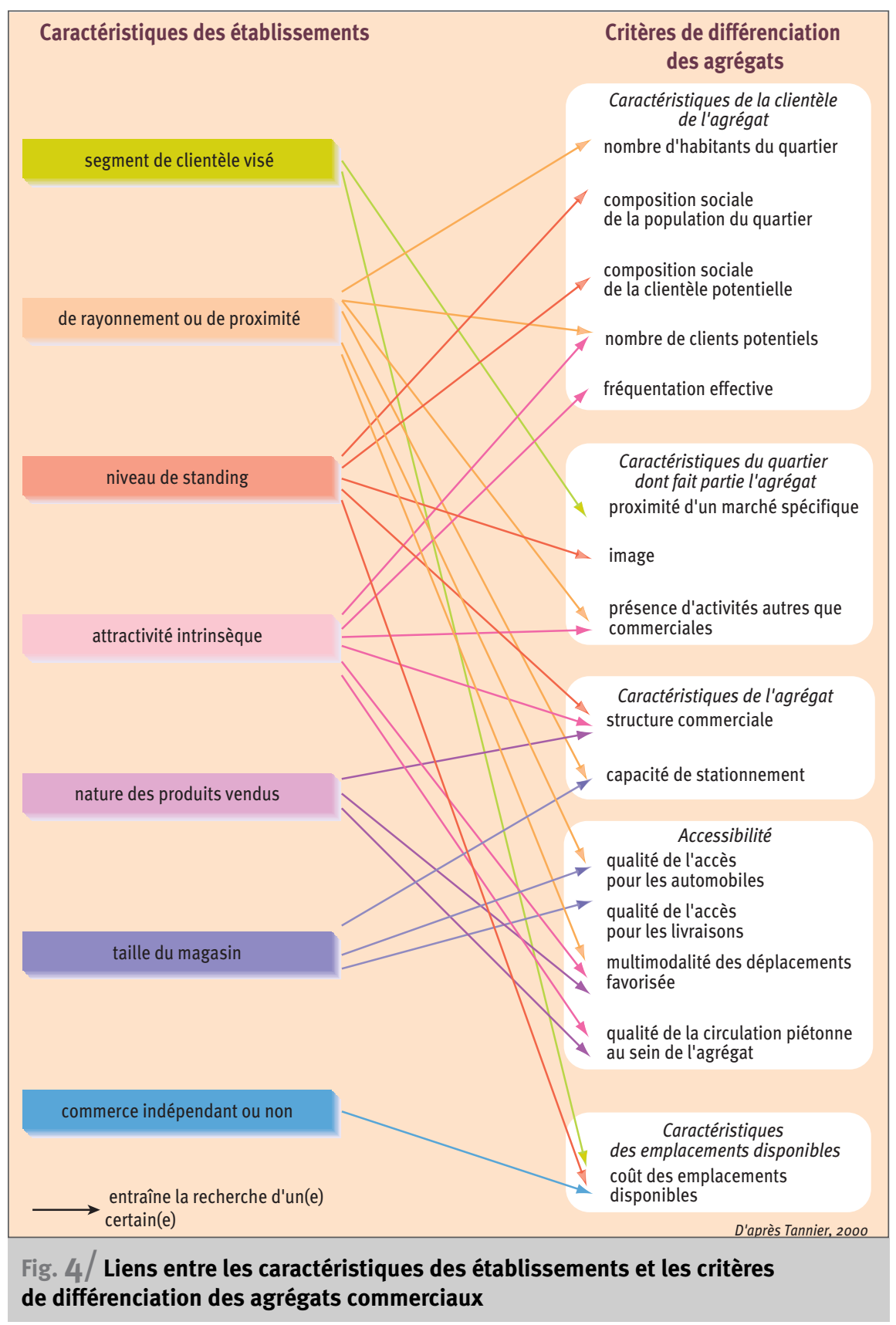

Finalement, les critères mentionnés sur la figure 4 permettent de différencier les agrégats commerciaux, du point de vue de leur attractivité sur les différents types d'établissements commerciaux.

Le passage de cette figure à la définition de règles d'implantation consiste en l'identification des modalités qualitatives de chaque critère, en fonction de chaque caractéristique des établissements. Prenons l'exemple de la figure 5. Un établissement commercial ayant pour cible une clientèle étudiante cherchera à trouver un agrégat situé à proximité d'une activité attirant un grand nombre d'étudiants (université notamment). On constate donc sur la figure une relation forte entre la caractéristique «segment de clientèle visé» et le critère "proximité d'un marché spécifique». Pour bénéficier de la présence d'un grand nombre d'étudiants, l'établissement sera prêt à payer assez cher son emplacement, ce qui explique la faible intensité de la relation entre la caractéristique de l'établissement "segment de clientèle visé: clientèle étudiante» et la modalité "peu élevé» du critère "coût des emplacements disponibles".

Cette cinquième figure présente l'ensemble des règles d'implantation relatives à l'une des caractéristiques des établissements commerciaux (le segment de clientèle visé), mais six autres groupes de règles ont été définis, correspondant chacun à l'une des six autres caractéristiques descriptives des établissements commerciaux.

Finalement, ce deuxième modèle est structurellement plus simple que le premier. Les rôles des acteurs, exprimés par des flèches de nature différente sur la figure 2 
(agit sur, influence...), ne sont plus différenciés: on considère d'un côté les phénomènes dont on doit tenir compte lors de la définition de règles d'implantation des commerces en ville, et de l'autre, les phénomènes qui influencent trop indirectement les choix de localisation des établissements commerciaux pour qu'ils méritent d'être considérés. Par ailleurs, les rétroactions directes et indirectes à l'ouverture d'établissements commerciaux (contrainte exercée par le territoire sur la figure 2) ne sont pas prises en compte.

Cependant, tandis que le premier modèle donne une vision globale, mais peu détaillée, de la question des localisations commerciales de détail en milieu urbain, le deuxième modèle analyse plus finement un phénomène, à savoir l'implantation des établissements commerciaux.

\section{Un troisième modèle pour évaluer l'attractivité des agrégats commerciaux d'une ville}

Le modèle a été conçu dans une optique de simulation. Il ne sert donc pas à calculer une seule et unique mesure d'attractivité réelle; il doit être utilisé pour comparer les attractivités obtenues, en fonction de conditions initiales différentes. Au regard du précédent modèle, les règles d'implantation sont simplifiées: on travaille dorénavant sur dix types d'établissements 5 , définis sur la base de quatre caractéristiques (rayonnement de l'établissement, standing, attractivité intrinsèque, taille du magasin).

Les principes généraux qui président à la réalisation du modèle sont les suivants. L'intensité de la relation entre une caractéristique descriptive d'un établissement et un critère de différenciation des agrégats représente l'importance, le poids de ce critère dans le processus d'évaluation. L'évaluation d'un critère par un type d'établissements correspond à la satisfaction de ce dernier vis-à-vis de la valeur dudit critère. Nous considérons une relation imprécise entre la valeur effective d'un critère et son évaluation par un type d'établissement. Nous cherchons à obtenir une mesure synthétique de l'évaluation de l'attractivité de chaque agrégat par chaque type d'établissements.

Grâce au modèle précédent, nous disposons d'éléments d'information sur l'intensité de la relation reliant les critères de différenciation des agrégats aux caractéristiques des établissements. Mais la quantification de cette relation n'a été qu'amorcée et nous devons poursuivre ce travail (fig. 5). En outre, nous ne connaissons que les modalités qualitatives des critères attendues par les établissements. Ainsi, la réalisation du troisième modèle suppose une nouvelle phase de recherche d'information.

La définition de règles d'évaluation de l'attractivité d'un agrégat commercial ne nécessitant pas le passage par l'agrégation de perceptions individuelles, nous avons choisi de nous baser sur des dires d'experts pour obtenir une quantification du lien entre les valeurs effectives des attributs et leur évaluation. Ces dires d'expert consistent en l'énoncé de constats généraux tels que: «En général, un petit établissement commercial de proximité est satisfait quand le quartier dans lequel s'insère l'agrégat commercial évalué compte au moins 4000 habitants." La quantification de l'importance des critères pour chaque type d'établissements est également obtenue à partir de dires d'experts.

Découlant de ces choix méthodologiques, le troisième modèle se présente ainsi 6 . Chaque agrégat commercial est caractérisé par une série d'attributs correspondant aux critères de différenciation des agrégats. Chaque type d'établissement est caractérisé par une série de valeurs représentant, pour chacun, l'importance (le poids) de chaque
5. Hypermarché, supermarché, supérette, petit établissement de proximité, petit établissement de rayonnement, maxi-discompte, grand magasin et magasin populaire, grande surface spécialisée, moyenne surface spécialisée et petit établissement haut de gamme.

6. Les principes présidant à la formalisation de ce troisième modèle ont été décrits de manière plus détaillée dans (Tannier, Frankhauser, 2001). 


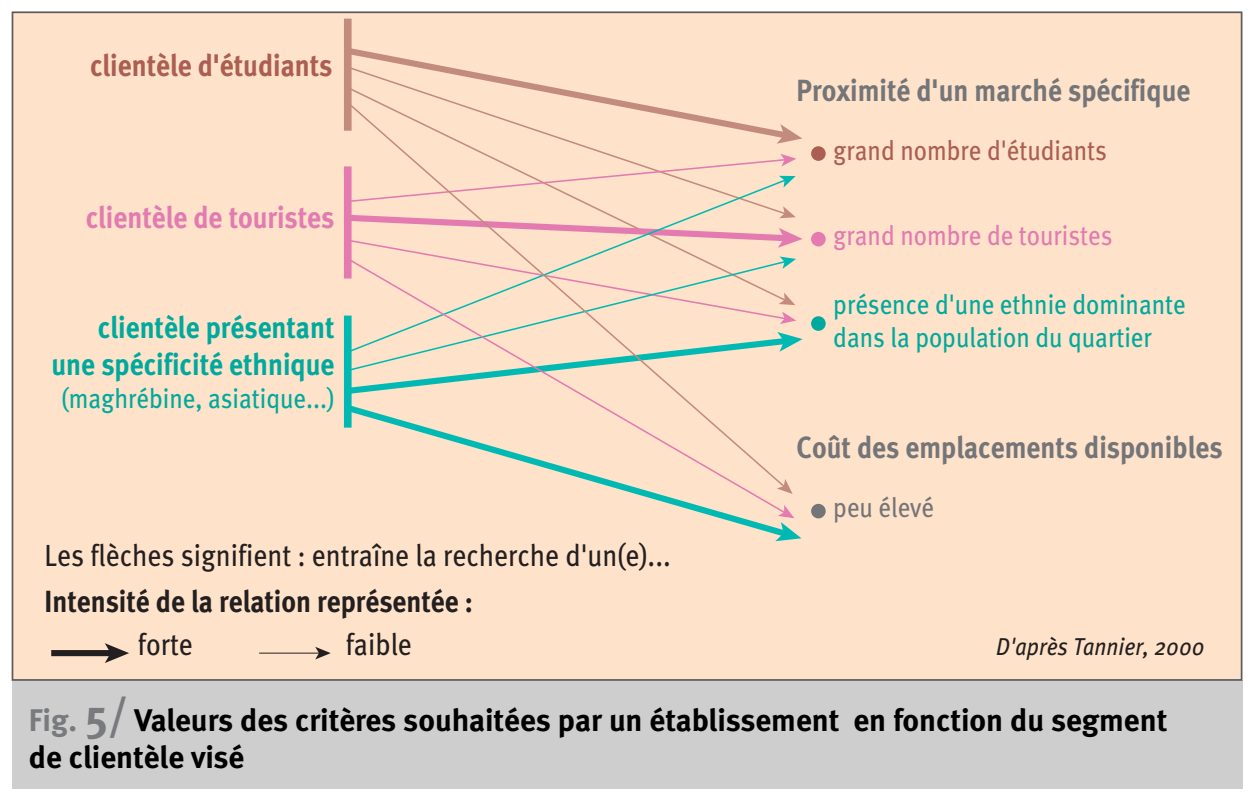

attribut lors de l'évaluation de l'attractivité d'un agrégat. Les règles définissant l'évaluation des attributs par chaque type d'établissements sont formalisées par l'intermédiaire de variables floues (fonctions mathématiques propres à la théorie des sous-ensembles flous). Un opérateur mathématique (la moyenne géométrique) permet d'agréger, en une valeur d'attractivité unique, tous les couples «évaluation d'un attribut-importance de cet attribut» caractérisant un agrégat pour un type d'établissement.

Une fois le modèle complètement spécifié, un premier ensemble de simulations a permis de l'ajuster. Des modifications successives lui ont été apportées (notamment afin de contrôler la sensibilité du modèle à de très faibles valeurs d'évaluation), qui ont permis d'améliorer nettement la qualité de représentation des phénomènes considérés. Puis, une nouvelle série de tests a été réalisée, au cours desquels on a fait subir de fortes variations aux valeurs des attributs. Les résultats ont montré que les variations de l'attractivité des agrégats, suite à la modification de leurs attributs, sont vraisemblables: l'amplitude de la réaction du modèle à la modification des attributs d'un agrégat correspond à l'amplitude des transformations susceptibles de se produire en réalité.

Ainsi, bien que présentant encore un certain nombre d'imperfections, le modèle représente de façon satisfaisante l'évaluation de l'attractivité des agrégats commerciaux d'une ville par différents types d'établissements. Ensuite, afin de mettre en évidence l'utilité pratique du modèle, nous l'avons appliqué pour répondre à deux questions susceptibles de se poser en matière d'aménagement commercial urbain. Ces deux applications sont inspirées de deux projets d'aménagement en cours de réalisation dans l'agglomération bisontine: la création d'un centre commercial d'envergure régionale dans le centre-ville et la restructuration d'un noyau commercial de proximité dans un quartier sensible, le quartier Brulard.

Création d'un nouveau centre commercial au centre-ville de Besançon

La principale source d'interrogation des décideurs publics concerne les risques de 
vacance de locaux inhérents au projet. $A$ priori, le modèle ne permet pas de lever cette interrogation car, dans le cas d'un agrégat de la taille d'un centre-ville, un centre commercial n'est qu'un élément parmi d'autres de la structure commerciale de l'agrégat. Cependant, une simulation réalisée à partir des attributs actuels du centre-ville bisontin montre que celui-ci est très attractif pour les moyennes surfaces spécialisées. Il se trouve que ces dernières ne s'y installent pas en raison du manque d'emplacements de taille suffisante disponibles. Ainsi, si le projet de centre commercial prévoit de réserver une grande partie de la surface commerciale à des emplacements de taille suffisante pour l'implantation de moyennes surfaces spécialisées, les risques de vacance de locaux seront minimisés.

\section{Amélioration de la structure commerciale d'un quartier sensible}

Le projet envisagé par la municipalité est de rénover et restructurer la galerie marchande du quartier, puis d'y installer un supermarché ou un maxi-discompte. L'hypothèse sous-jacente au projet est que la restructuration de la galerie commerciale va améliorer l'image du quartier. Comme il est également prévu d'agrandir le parking de 15 à 50 places, l'agrégat devrait devenir suffisamment attractif pour qu'un supermarché ou un maxi-discompte vienne s'y installer.

Une première série de simulations montre que, suite aux améliorations prévues, l'attractivité de l'agrégat par un maxi-discompte augmentera légèrement, tout en demeurant moyenne. Pour un supermarché, l'attractivité, actuellement très faible, deviendra faible. Il apparaît par ailleurs que l'accessibilité de cet agrégat est actuellement très mauvaise et la restructuration de la galerie marchande ne permettra pas de changer cet état de fait. L'augmentation de la capacité de stationnement représente, elle, un aspect positif du projet envisagé; mais elle devra être accompagnée d'une amélioration de l'accessibilité pour que l'agrégat devienne réellement attractif vis-à-vis de l'un ou l'autre des deux types d'établissements envisagés. Ainsi, dans l'hypothèse d'une évaluation de l'attribut "accessibilité» nettement améliorée pour les deux types d'établissements, l'évaluation de l'attractivité de l'agrégat deviendrait assez bonne pour un maxi-discompte et moyenne pour un supermarché. On pourrait alors raisonnablement espérer qu'un maxi-discompte désire s'implanter dans l'agrégat, mais pas un supermarché.

\section{Conclusion}

Actuellement, un des points "phares» de la recherche géographique dans le domaine de la modélisation et de la simulation est le couplage de modèles, terme qui sous-entend l'intégration plus ou moins poussée de différents outils de simulation (systèmes d'information géographique, systèmes multi-agents, systèmes experts...), afin de tirer parti des avantages respectifs de chacun de ces outils. Mais pour améliorer l'efficacité et la pertinence des modèles de simulation que nous construisons, il peut être aussi très profitable de chercher à mettre en œuvre, à l'amont, des outils conceptuels adaptés.

Ainsi, les deux premiers modèles que nous avons décrits représentent une aide à la conception d'un modèle de simulation. Leur construction a soulevé de nombreuses interrogations, parmi lesquelles certaines auraient été éludées si le troisième modèle avait été directement conçu. La succession de plusieurs étapes dans la réflexion a eu 
pour effet d'éclaircir les hypothèses à la base de ce dernier modèle, d'en justifier la structure et la formalisation. Il est en outre relativement aisé d'introduire dans chacun des trois modèles de nouvelles connaissances sur le thème de la localisation des établissements commerciaux en milieu urbain. Basé sur les deux premiers, le troisième modèle apparaît finalement moins figé, moins lié à un état de la connaissance géographique, que ne le sont de nombreux autres modèles de simulation.

D'une certaine manière, on peut mettre en parallèle l'enchaînement des trois modèles que nous avons présentés et la succession des étapes qui existent dans les méthodes Merise ou UML (Unified Modelling Language)... Ce parallèle est toutefois très relatif car ces deux méthodes sont des outils conceptuels permettant de modéliser la structure et le fonctionnement d'une infinité de systèmes, tandis que la démarche

\section{Références}

qui a fait l'objet de cet article reste spécifique à un type de problème.

Agences d'urbanisme de Belfort et de Montbéliard (1997). Commerce et modes de ville, Actes des $18^{\mathrm{e}}$ Rencontres des agences d'urbanisme, FNAU (Fédération nationale des agences d'urbanisme), $296 \mathrm{p}$.

Brown S. (1992). Retail location: a micro-scale perspective. Aldershot Hants: Avebury, 315 p.

COLLETIS-WAHL K. (1995). "L'hypothèse des facteurs de concurrence spatiale, quels fondements?». Revue d'économie régionale et urbaine, $\mathrm{n}^{\circ} 5, \mathrm{p} .803-824$.

DuRAND-DASTÈs F. (1984). "Systèmes et localisations: problèmes théoriques et formels». Géopoint 84 : systèmes et localisations. Avignon: Groupe Dupont, p. 19-44.

GACÔGNE L. (1997). Éléments de logique floue. Paris: Hermès, 253 p.

LE BERRE M. (1987a). «Itinéraire géographique, 20 ans après ». Brouillons Dupont, nº 17, $115 \mathrm{p}$.

Le BerRe M. (1987b). De l'induction à la modélisation systémique en géographie. Besançon: Université, thèse d'État, $560 \mathrm{p}$.

Metton A. (1984). «Mutations commerciales et milieux urbains », in Le Commerce urbain français, présenté par A. Metton, avant-propos de J. Beaujeu-Garnier. Paris: Presses universitaires de France, coll. "Université d'Orléans », p. 9-94.

JONES K., Simmons J. (1990). Location, location, location. Analysing the retail environment. Scarborough, Ontario: Nelson Canada, 438 p.

PAulRÉ B. (1985). La Causalité en économie. Signification et portée de la modélisation structurelle. Lyon: Presses universitaires de Lyon, $440 \mathrm{p}$.

PÉRON R. (1980). Contribution à l'analyse des rapports entre urbanisation et restructuration de l'appareil commercial de détail, le cas de Rennes. Rennes: Université de Rennes II, thèse, $250 \mathrm{p}$.

Scom P. (1973). Geography and retailing. Londres: Hutchinson University Library, 192 p.

Simmons J. (1964). The Changing Pattern of Retail Location. Chicago: Univ. of Chicago, Department of geography, Research paper $n^{\circ} 92,202 \mathrm{p}$.

TANNIER C. (2000). Les Localisations commerciales de détail en milieu urbain. Mieux connaître, par la modélisation, pour mieux aménager. Besançon: Université de Franche-Comté, thèse de géographie, sous la direction de M. Le Berre, $334 \mathrm{p}$.

TANnier C., Frankhauser P. (2001). «From the observations to the construction of an urban dynamics simulation model: an inductive approach ». Cybergeo, $n^{\circ} 191,18$ p., http://www.cybergeo.presse.fr/modelis/tannier/tannier.htm 